\title{
Curvature effects on spiral spectra: Generation of point eigenvalues near branch points
}

\author{
Björn Sandstede* \\ Department of Mathematics and Statistics, University of Surrey, Guildford, GU2 7XH, UK \\ Arnd Scheel ${ }^{\dagger}$ \\ School of Mathematics, University of Minnesota, Minneapolis, MN 55455, USA
}

(Dated: October 7, 2005)

\begin{abstract}
It is shown that spiral waves may possess many isolated point eigenvalues that appear near branch points of the linear dispersion relation. These eigenvalues are created by the same mechanism that leads to infinitely many bound states for selfadjoint Schrödinger operators with sufficiently weakly decaying long-range potentials. For spirals, the weak decay of the potential is due to curvature effects on the profile of the spiral in an intermediate spatial range that separates the spiral core from the far field.
\end{abstract}

PACS numbers: 82.40.Ck, 05.45.-a, 47.54.+r

\section{INTRODUCTION}

Spiral waves have been observed in various biological, chemical and physical systems [1], as well as in numerical simulations of reaction-diffusion systems and complex Ginzburg-Landau equations. Part of their fascination is due to the intriguing instabilities they exhibit: For instance, spirals may begin to meander or to drift, a scenario that has been observed in the BelousovZhabotinsky reaction [2], during fibrillations in cardiac tissue [3], and in the oxidation of carbon-monoxide on platinum surfaces [4], and that has been attributed to a supercritical Hopf bifurcation [5]. Of particular relevance to this paper is spiral breakup where the core [6] or the far field $[7,8]$ of a spiral wave breaks up into a turbulent region with complex spatio-temporal behavior. Other interesting instabilities are spatio-temporal period-doubling bifurcations, which have been observed in the Belousov-Zhabotinsky reaction [9] and in numerical simulations [10], and transverse instabilities [11] that are characterized by a degenerate dispersion relation between asymptotic wavelength and wave speed.

Among the challenges for theoretical studies of spirals are the tasks of investigating the nature of the above instabilities and making predictions about the patterns emerging from them. The first step toward these goals is to understand spiral spectra as they inform us about the stability or instability of a spiral and, through the associated eigenmodes, about the spatio-temporal behavior associated with unstable modes. Spiral spectra are composed of two disjoint sets: a discrete part consisting of isolated point eigenvalues, and a continuous part consisting of curves (for planar spirals) or of densely distributed eigenvalues (for spirals on large disks). Isolated point eigenvalues depend genuinely on the spiral in

\footnotetext{
*Electronic address: b.sandstede@surrey.ac.uk

${ }^{\dagger}$ Electronic address: scheel@math.umn.edu
}

the sense that their location is determined by the spiral shape between core and far field; in general, their computation therefore requires knowledge of the entire two-dimensional spiral. In contrast, the continuous part of the spectrum depends only on the asymptotic onedimensional profile of the spiral in the far field. It is also known, for instance from Ref. [12], that point eigenvalues and absolute instabilities caused by the continuous part of the spectrum produce very different scaling laws and bifurcation diagrams near onset. It is therefore desirable to develop analytical criteria that allow us to decide whether spirals destabilize due to discrete or continuous parts of their spectrum.

In this paper, we show that spiral spectra may contain many isolated point eigenvalues whose approximate location can be predicted from the asymptotic wave trains upon accounting properly for curvature effects in the region between core and far field. Specifically, we show that the linearization about a planar spiral wave near double roots (or branch points) of its linear dispersion relation can be reduced to a one-dimensional Schrödinger operator with a complex long-range potential. Depending on a sign condition on its coefficients, this Schrödinger operator has infinitely many bound states which correspond to isolated point eigenvalues of the spiral. These eigenvalues can shift the onset to instability, and our result predicts precisely when such a shift occurs.

We apply our analyses to a modified FitzHughNagumo equation and the complex Ginzburg-Landau equation: Our theoretical results turn out to be in excellent agreement with the recent spectral computations by Barkley and Wheeler [13] for the modified FitzHughNagumo equation, which in fact motivated our study. Furthermore, we show that spiral waves in the complex Ginzburg-Landau equation can possess discrete eigenvalues generated by curvature effects and that these eigenvalues lead to a shift of the onset of spiral instability into the regime where the asymptotic wave trains are only convectively unstable. 


\section{SPIRAL SPECTRA}

To set the scene, we assume that $u_{*}(r, \varphi-\omega t)$, written in polar coordinates, is a rigidly-rotating Archimedean spiral wave with nonzero angular velocity $\omega$ of the reaction-diffusion system

$$
u_{t}=D \Delta u+f(u), \quad u \in \mathbb{R}^{n},
$$

where $(x, y)$ lies in $\mathbb{R}^{2}$ or in large disks $B_{R}(0)$ of radius $R \gg 1$ with, say, Neumann boundary conditions. We assume that the diffusion matrix $D$ is invertible but emphasize that our analysis applies, under certain conditions, to systems for which some of the diffusion coefficients vanish (see Eq. (20) below for an example). A typical spiral wave is shown in Fig. 1. In particular, the asymptotic profile of a spiral in the far field away from its core is locally of the form $u_{0}(\kappa r+\varphi-\omega t)$, where $u_{0}$ is $2 \pi$-periodic in its argument; we refer to Eq. (7)-(8) for the precise far-field asymptotics of $u_{*}$. The function $u_{0}(\kappa x-\omega t)$ is a planar wave that satisfies Eq. (1) on $\mathbb{R}^{2}$. Linearizing Eq. (1) about this planar wave gives

$$
w_{t}=D \Delta w+f_{u}\left(u_{0}(\kappa x-\omega t)\right) w, \quad(x, y) \in \mathbb{R}^{2} .
$$

In the following, we focus on longitudinal instabilities of the wave train which are captured by the ansatz

$$
w=\mathrm{e}^{\lambda t+\nu x} w_{0}(\kappa x-\omega t ; \nu)
$$

where $\lambda, \nu \in \mathbb{C}$ and $w_{0}(\vartheta ; \nu)$ is $2 \pi$-periodic in $\vartheta$. Substitution into Eq. (2) yields the linear dispersion relation $\lambda_{*}(\nu)$ of the wave train in the propagation direction and its group velocity $c_{\mathrm{g}}=-\mathrm{d} \lambda_{*} /\left.\mathrm{d} \nu\right|_{\nu=0} \in \mathbb{R}$ in the laboratory frame. As we shall see below, the linear dispersion relation in the transverse direction is not relevant to our spectral analysis, but will nevertheless affect the spatial spiral asymptotics given in Eq. (7)-(8) below. We therefore also introduce the ansatz

$$
w=\mathrm{e}^{\lambda t+\nu y} w_{0}^{\perp}(\kappa x-\omega t ; \nu)
$$

with $\lambda, \nu \in \mathbb{C}$ and $w_{0}^{\perp}(\vartheta ; \nu)$ being $2 \pi$-periodic in $\vartheta$, to get the linear dispersion relation $\lambda_{*}^{\perp}(\nu)=d_{\perp} \nu^{2}+\mathrm{O}\left(\nu^{3}\right)$ in the transverse direction.

To relate these quantities to the spectrum of the spiral, we shall work in the corotating frame $\varphi \mapsto \varphi+\omega t$ in which Eq. (1) becomes

$$
u_{t}=D \Delta u+\omega \partial_{\varphi} u+f(u) .
$$

The eigenvalue problem associated with the spiral $u_{*}$ is then given by

$$
\lambda u=D \Delta u+\omega \partial_{\varphi} u+f_{u}\left(u_{*}(r, \varphi)\right) u .
$$

We now briefly summarize the results established in Refs. [14, 15]. Throughout, we reserve the term point eigenvalue to denote eigenvalues with finite multiplicity that are isolated uniformly in the disk radius $R$.
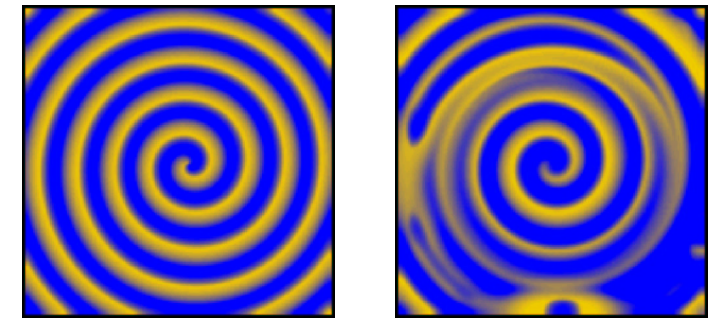

FIG. 1: Contour plots of 2D spiral waves of the FitzHughNagumo equation (20) computed on squares with Neumann boundary conditions are shown. The left plot shows a rigidly rotating spiral, while the right plot shows a spiral that is breaking up in the far field.

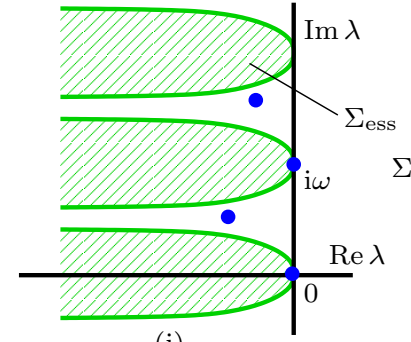

(i)

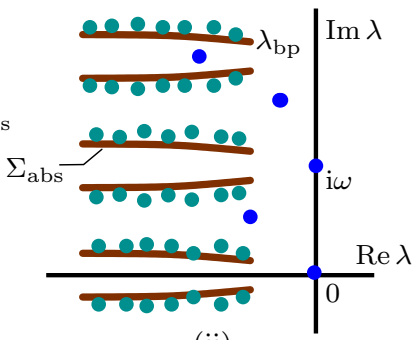

(ii)
FIG. 2: (i) Spectra of spirals on $\mathbb{R}^{2}$. (ii) Spectra of spirals on disks $B_{R}(0)$ with $R \gg 1$. See text for details.

On $\mathbb{R}^{2}$, spiral spectra consist of point eigenvalues, among them eigenvalues at 0 and $\pm \mathrm{i} \omega$ which are enforced by the rotation and translation symmetry of the plane, and the essential spectrum $\Sigma_{\text {ess }}$, which is bounded by the linear dispersion curves $\lambda=\lambda_{*}(\mathrm{i} \gamma)+\mathrm{i} \omega \ell$ with $\gamma \in \mathbb{R}$ and $\ell \in \mathbb{Z}$ (see Fig. 2(i)). The vertical periodicity with period $\omega$ of the essential spectrum in the complex plane arises since the essential spectrum is determined entirely by the limiting eigenvalue problem for $r \rightarrow \infty$ : In this limit, the diffusion operator $\Delta$ is replaced by $\partial_{r r}$, and the eigenvalue problem (5) acquires an additional symmetry due to the term $\omega \partial_{\varphi}$ which acts by replacing eigenmodes $w$ and spectrum $\lambda$ by $w \exp (\mathrm{i} \ell \varphi)$ and $\lambda+\mathrm{i} \omega \ell$, respectively.

On the other hand, on disks $B_{R}(0)$ of radius $R$, spiral spectra converge as $R \rightarrow \infty$ to the union of the absolute spectrum $\Sigma_{\text {abs }}$ and point eigenvalues (see Fig. 2(ii)). The absolute spectrum consists of all elements $\lambda \in \mathbb{C}$ for which the equation $\lambda=\lambda_{*}(\nu)+\mathrm{i} \omega \ell$ has two roots $\nu_{1}$ and $\nu_{2}$ with equal real part that have the correct Morse index (see Refs. [14, 15] for details). It is therefore determined solely by the asymptotic one-dimensional wave trains and again vertically periodic in the complex $\lambda$-plane with period $\omega$. The edges of the absolute spectrum are given by branch points $\lambda_{\mathrm{bp}}$ of the linear dispersion relation where, by definition, the two roots $\nu_{1}$ and $\nu_{2}$ become equal to each other to form a double root of $\lambda_{*}(\nu)$ : In other words, $\lambda_{\text {bp }}=\lambda_{*}\left(\nu_{1}\right)+\mathrm{i} \omega \ell$ and $\mathrm{d} \lambda_{*} / \mathrm{d} \nu\left(\nu_{1}\right)=0$. The absolute spectrum itself is not spectrum, but each of its elements is approximated by infinitely many eigenvalues of (5) as $R \rightarrow \infty$; the convergence toward the absolute spectrum 


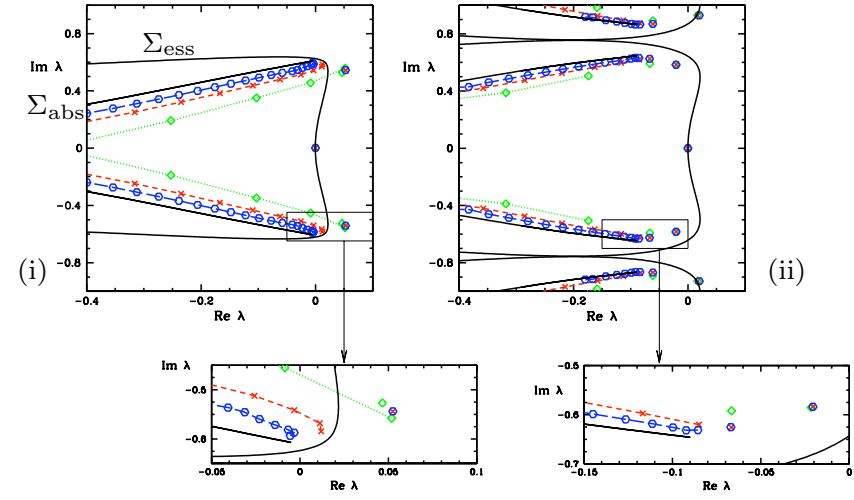

FIG. 3: Spiral spectra computed by Barkley and Wheeler [13] for the FitzHugh-Nagumo equation (20) near core (i) and farfield (ii) breakup on disks of radius $R=20,40,80$, indicated by different symbols and colors [Reproduced with permission]. The absolute and essential spectra shown in the above plots were previously computed in Ref. [14].

is algebraic of order $1 / R$ in $R$. While the absolute spectrum does not depend on the boundary conditions, the actual eigenvalues near it will. The point eigenvalues, which make up the remaining part of the spectrum, have limits as $R \rightarrow \infty$, which are approached exponentially fast of order $\exp (-\alpha R)$ in $R$, and these limiting elements contain all eigenvalues and resonance poles of the spiral on $\mathbb{R}^{2}$ plus possibly additional eigenvalues induced by the boundary conditions.

The theoretical predictions for spiral spectra on large disks were recently corroborated by numerical computations, summarized in Fig. 3, by Barkley and Wheeler [13], which confirm in particular the clustering of eigenvalues along the absolute spectrum. In addition, however, their computations show that certain point eigenvalues appear to be intimately linked to the absolute spectrum by aligning themselves along rays that emerge from the edges of the absolute spectrum. As visible in Fig. 3(ii), this phenomenon seems to occur along each branch of the absolute spectrum (recall that the absolute spectrum is vertically periodic with period $\omega$ ). These observations are surprising as point eigenvalues are determined by the shape of the spiral in the region between core and far field rather than by the far field itself.

In this paper, we explain the occurrence of these point eigenvalues in terms of curvature corrections to the spiral shape in an intermediate spatial regime between core and far field which manifest themselves as a long-range potential in Eq. (5). Our analysis provides criteria which determine whether instabilities such as core or far-field breakup are caused by the absolute spectrum or by point eigenvalues arising through curvature effects. We shall focus on spirals on $\mathbb{R}^{2}$ since point eigenvalues persist on disks of finite but large radius $R$, independently of the boundary conditions.

\section{REDUCTION TO SCHRÖDINGER EQUATIONS}

We now reduce the eigenvalue problem of a planar spiral wave near branch points to a non-selfadjoint Schrödinger operator with a complex long-range potential. The result is given toward the end of this section in Eq. (13).

It will be convenient to write the steady-state equation for Eq. (4) on $\mathbb{R}^{2}$ in polar coordinates:

$$
D\left[u_{r r}+\frac{u_{r}}{r}+\frac{u_{\varphi \varphi}}{r^{2}}\right]+\omega u_{\varphi}+f(u)=0 .
$$

To capture point eigenvalues, we need to expand the spiral wave in the far field as $r \rightarrow \infty$. Using the Archimedean coordinate

$$
\vartheta=\varphi+\kappa r+\theta_{1} \log r
$$

where the constant $\theta_{1}$ is to be determined, the spiral wave $u_{*}(r, \vartheta)$ has, for sufficiently large $r \gg 1$, the expansion

$$
\begin{aligned}
u_{*}(r, \vartheta) & =u_{0}(\vartheta)+\frac{1}{r} u_{1}(\vartheta)+\mathrm{O}\left(1 / r^{2}\right), \\
u_{1}(\vartheta) & =b u_{0}^{\prime}(\vartheta)+\theta_{1} \partial_{\kappa} u_{0}(\vartheta)+\frac{\kappa}{2} \partial_{\nu \nu} w_{0}^{\perp}(\vartheta ; 0) .
\end{aligned}
$$

Here, $\theta_{1}=\kappa d_{\perp} / c_{\mathrm{g}}, w_{0}^{\perp}$ has been introduced in Eq. (3), $\partial_{\kappa} u_{0}$ refers to the derivative of the wave train profile with respect to its spatial wave number $\kappa$, and $b \in \mathbb{R}$ is a constant that is determined by a compatibility condition at order $1 / r^{2}$. The expansion (8) can be verified by substituting it into Eq. (6) and expanding in powers of $1 / r$. Writing the eigenvalue problem (5) as a first-order system in the Archimedean variables, we obtain

$$
\begin{aligned}
u_{r}= & -\left(\kappa+\frac{\theta_{1}}{r}\right) u_{\vartheta}+v \\
v_{r}= & -\frac{u_{\vartheta \vartheta}}{r^{2}}+D^{-1}\left[\lambda-\omega \partial_{\vartheta}-f_{u}\left(u_{*}(r, \vartheta)\right)\right] u \\
& -\left(\kappa+\frac{\theta_{1}}{r}\right) v_{\vartheta}-\frac{v}{r},
\end{aligned}
$$

which, using the expansion (8), is of the form

$$
U_{r}=\left[A_{0}(\lambda)+\frac{1}{r} A_{1}(\lambda)+\mathrm{O}\left(\frac{1}{r^{2}}\right)\right] U, \quad U=\left(\begin{array}{c}
u \\
v
\end{array}\right),
$$

where the operators $A_{j}$ act on functions $U=(u, v)$ that are $2 \pi$-periodic in $\vartheta$. Since all eigenmodes are necessarily smooth in the $\vartheta$-direction, the $u_{\vartheta \vartheta} / r^{2}$ terms in Eq. (9) can be considered as higher-order corrections that do not cause any regularity problems.

Upon taking the limit $r \rightarrow \infty$ in Eq. (9)-(10), we recover the eigenvalue problem of the asymptotic wave train. In particular, a direct computation shows that $\nu$ is an eigenvalue of $A_{0}(\lambda)$, given by

$$
A_{0}(\lambda)=\left(\begin{array}{cc}
-\kappa \partial_{\vartheta} & 1 \\
D^{-1}\left[\lambda-\omega \partial_{\vartheta}-f_{u}\left(u_{0}(\vartheta)\right)\right] & -\kappa \partial_{\vartheta}
\end{array}\right),
$$


if, and only if, it is a root of the linear dispersion of the asymptotic wave train, i.e. if $\lambda$ and $\nu$ are related via $\lambda=\lambda_{*}(\nu)$.

Suppose now that $\lambda_{\text {bp }} \in \mathbb{C}$ is a branch point of the linear dispersion relation $\lambda_{*}(\nu)$ of the spiral wave with double roots $\nu_{1}=\nu_{2}$. Interpreted in the context of Eq. (10), this assumption means that $A_{0}\left(\lambda_{\mathrm{bp}}\right)$ has a double eigenvalue $\nu_{1}=\nu_{2}$. We shall assume that the double eigenvalue $\nu_{1,2}$ has geometric multiplicity one, which is the generic case.

Our goal is now to reduce Eq. (10) to a simpler scalar equation for $\lambda$ near $\lambda_{\mathrm{bp}}$. Thus, we employ the perturbation ansatz

$$
U(r)=\mathrm{e}^{\nu_{1} r} V(r), \quad \lambda=\lambda_{\mathrm{bp}}+\Lambda, \quad \nu=\nu_{1}+\mathcal{V},
$$

so that Eq. (10) becomes

$$
V_{r}=\left[A_{0}-\nu_{1}+\frac{1}{r} A_{1}+\mathrm{O}\left(\frac{1}{r^{2}}\right)\right] V
$$

where $A_{0}-\nu_{1}$ evaluated at $\Lambda=0$ now has a double eigenvalue at $\mathcal{V}=0$ with geometric multiplicity one. In particular, we can apply center-manifold theory to Eq. (11) for $\Lambda$ and $1 / r$ close to zero, which shows that we can reduce Eq. (11) to an equation of the form

$$
W_{r}=\left[\left(\begin{array}{cc}
0 & 1 \\
\Lambda / d & 0
\end{array}\right)+\frac{1}{r} B_{1}+\mathrm{O}\left(\frac{1}{r^{2}}\right)\right] W
$$

where $W=\left(W_{1}, W_{2}\right) \in \mathbb{C}^{2}$, for a certain complex coefficient $d \in \mathbb{C}$ and a complex $2 \times 2$ matrix $B_{1}$. The components $W_{1}$ and $W_{2}$ of $W$ can be thought of as being the amplitudes of the eigenmode and the generalized eigenmode, respectively, associated with the double eigenvalue $\mathcal{V}=0$ of the right-hand side of Eq. (11) at $\Lambda=0$.

Equation (12) is equivalent to a second-order equation for $W_{1}$. Upon removing the $\partial_{r} W_{1}$ term in this secondorder equation by an appropriate coordinate transformation, we finally arrive at the scalar non-selfadjoint Schrödinger equation

$$
d w_{r r}+\left[\frac{a}{r}+\mathrm{O}\left(\frac{1}{r^{2}}\right)\right] w=\Lambda w, \quad r \in\left[R_{0}, \infty\right)
$$

for the variable $w$, which corresponds to the transformed $W_{1}$. Here, $R_{0} \gg 1$ is determined by the region of validity of the center-manifold reduction, while the coefficient $a \in \mathbb{C}$ describing the strength of the complex long-range potential in Eq. (13) is determined from the entries of the matrix $B_{1}$ in Eq. (12).

Equation (13) describes the eigenvalue problem of the spiral wave in the far field. To obtain genuine eigenmodes of the spiral wave, we need to match its solutions to the eigenvalue problem in the core region. We have carried out this matching process in Ref. [15] for fronts in reaction-diffusion equations and summarized the necessary modifications for spirals in Ref. [14]. Therefore, we shall omit the details here. The result of the matching with the core is an effective boundary condition for Eq. (13) at $r=R_{0}$ of the form

$$
s_{1}(\Lambda) w\left(R_{0}\right)+s_{2}(\Lambda) w_{r}\left(R_{0}\right)=0
$$

which provides the correct coupling to the eigenvalue problem near the spiral core. The complex-valued functions $s_{1}$ and $s_{2}$ depend smoothly on $\Lambda$, and satisfy $\left|s_{1}\right|^{2}+\left|s_{2}\right|^{2} \neq 0$ near $\Lambda=0$.

In summary, to determine the spectrum of the spiral wave near the branch point $\lambda_{\mathrm{bp}}$, it suffices to find the spectrum of the Schrödinger operator given in Eq. (13) subject to the boundary condition (14). Before turning to the point spectrum of (13)-(14), we shall discuss the interpretation of the coefficients $a$ and $d$.

The continuous spectrum of (13) is given by the ray $\Lambda=-d \gamma^{2}$ with $\gamma \in \mathbb{R}$ as it is determined entirely by the limiting problem at $r=\infty$. By construction, this spectrum must coincide with the absolute spectrum of the spiral wave near the branch point $\lambda_{\mathrm{bp}}$. The algorithm for the computation of the absolute spectrum of spirals outlined in Ref. [14] therefore allows us to calculate $d$ numerically.

The coefficient $a$ is determined as follows. Since $\mathrm{d}(1 / r) / \mathrm{d} r=\mathrm{O}\left(1 / r^{2}\right)$, we can treat the variable $1 / r$ in Eq. (11) for $r \gg 1$ as a slowly varying parameter. In particular, to compute the reduced vector field on the center manifold of Eq. (11) to leading order, we can simply consider $1 / r$ as a small parameter, rather than treating Eq. (11) as a genuinely non-autonomous system. This observation allows us to make the following argument. Truncating Eq. (13) at order $1 / r$ and rewriting the resulting equation as a first-order system yields

$$
\tilde{W}_{r}=\left(\begin{array}{cc}
0 & 1 \\
\frac{1}{d}\left(\Lambda-\frac{a}{r}\right) & 0
\end{array}\right) \tilde{W}, \quad \tilde{W}=\left(w, w_{r}\right) .
$$

The coefficient matrix has a double eigenvalue precisely when $\Lambda=a / r$. Thus, upon reversing the coordinate transformations that led from Eq. (9) to Eq. (15), we see that the coefficient matrix on the right-hand side of Eq. (9), also truncated formally at order $1 / r$, has a double eigenvalue precisely when $\lambda$ is equal to $\lambda_{\mathrm{bp}}+a / r$. Since the truncated coefficient matrix, acting on $2 \pi$-periodic functions in $\vartheta$, is explicitly given by

$$
\begin{aligned}
& A_{0}(\lambda)+\frac{1}{r} A_{1}(\lambda)= \\
& \left(\begin{array}{c}
1 \\
D^{-1}\left[\lambda-\omega \partial_{\vartheta}-f_{u}\left(u_{0}(\vartheta)+u_{1}(\vartheta) / r\right)\right]-\left(\kappa+\frac{\theta_{1}}{r}\right) \partial_{\vartheta}
\end{array}\right),
\end{aligned}
$$

and since we assumed that we know $\lambda_{\mathrm{bp}}$, we can therefore compute the value of $\lambda$ for which $A_{0}+A_{1} / r$ has a double eigenvalue numerically as a function of $1 / r$; from these values and the expansion $\lambda=\lambda_{\mathrm{bp}}+a / r$, we then obtain the coefficient $a$. 


\section{SPECTRA OF NON-SELFADJOINT SCHRÖDINGER EQUATIONS}

We now discuss the spectrum of (13)-(14). When

$$
\Phi:=\arg \left(\frac{a}{d}\right)
$$

satisfies $|\Phi|<\pi / 2$, the Schrödinger equation (13)-(14) has infinitely many point eigenvalues, given approximately by

$$
\Lambda_{n}=\frac{a^{2}}{4 d n^{2}}\left[1+\mathrm{O}\left(\frac{1}{n}\right)\right], \quad n \gg 1, \quad n \in \mathbb{N}
$$

that accumulate at the origin along the ray

$$
\arg \Lambda=\Psi=\arg \left(\frac{a^{2}}{d}\right)
$$

(see Fig. 4(i)). The point eigenvalues depend on the boundary conditions (14) only through the higher-order term $\mathrm{O}(1 / n)$. In contrast, when $|\Phi|>\pi / 2$, then (13)(14) does not have any point eigenvalues near the origin. At the transition point $\Phi= \pm \pi / 2$, the eigenvalues disappear through the essential spectrum by moving to the "wrong" Riemann sheet of the dispersion relation, thus corresponding to resonance poles with exponentially increasing eigenfunctions. Before we continue our discussion, we remark that both cases $|\Phi|<\pi / 2$ and $|\Phi|>\pi / 2$ do occur, and we refer to $\S \mathrm{V}$ below for examples.

We now provide arguments to support our claims. If the ratios $a / d$ and $s_{1} / s_{2}$ are real, then (13)-(14) is selfadjoint, and [16, Thm. XIII.6(a)] readily shows that the above dichotomy holds true. In the complex nonselfadjoint case, consider first the truncated Schrödinger equation

$$
w_{r r}+\frac{a}{d r} w=\frac{\gamma^{2}}{4} w, \quad \frac{\gamma^{2}}{4}=\frac{\Lambda}{d}, \quad r \in\left[R_{0}, \infty\right) .
$$

Its unique bounded solution $w(r)$ is the Whittaker function $W_{a /(d \gamma), 1 / 2}(\gamma r)$ which, according to Ref. [17, (4.4.18) and (4.4.33)], admits the expansion

$$
w\left(R_{0}\right)=R_{0}^{1 / 4} \cos \left(\sqrt{\frac{4 a R_{0}}{d}}-\frac{a \pi}{\gamma d}+\frac{\pi}{4}\right)\left[1+\mathrm{O}\left(|\gamma|^{1 / 2}\right)\right]
$$

at $r=R_{0}$. Substituting $w\left(R_{0}\right)$ and its derivative $w_{r}\left(R_{0}\right)$ into the boundary condition (14), we see that Eq. (14) is met provided $\gamma=\gamma_{n}$ where $\gamma_{n}=\frac{a}{n d}+O\left(1 / n^{2}\right)$ for integers $n \gg 1$. Note that the actual values of $s_{1}$ and $s_{2}$ enter only at higher order. Reverting back to the variable $\Lambda$, we obtain the asymptotics (17), as claimed. Lastly, using variation-of-parameters and [18, Thm. 11.1 in $\S 6]$ for the rescaled spatial variable $s=|\gamma| r$ in the limit $\gamma \rightarrow 0$, this analysis can be extended to the full problem (13) provided the coefficients in Eq. (13) are analytic in $r$ (the arguments in Ref. [18] require a holomorphic extension

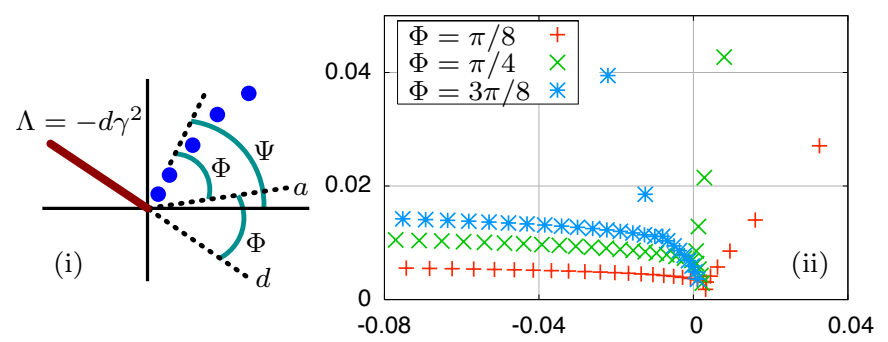

FIG. 4: The complex $\Lambda$-plane is shown: (i) When $\Phi=$ $\arg (a / d)$ satisfies $|\Phi|<\frac{\pi}{2}$, the Schrödinger equation (13) has infinitely many bound states that accumulate on the origin along the ray $\arg \Lambda=\Psi=\arg \left(a^{2} / d\right)$. (ii) Numerically computed spectra of (19) for $d=1$ and different values of $\Phi$ on the interval $(10,210)$ with Dirichlet and Neumann conditions at the left and right endpoints, respectively. Point eigenvalues accumulate approximately along the ray $\arg \Lambda=2 \Phi$.

to complex $r$ ). We do not currently have mathematical proofs in the case of non-analytic coefficients, but remark that all numerical simulations with non-analytic coefficients that we performed have produced results consistent with the claimed alignment with $\arg \Lambda=\Psi$.

Hence, the results derived in this section indicate that spiral waves will have many point eigenvalues to the right of branch points of the linear dispersion relation whenever the angles $\Phi$ and $\Psi$ are smaller than $\pi / 2$.

One standard criterion for the onset of absolute instability of spirals is the saddle-point condition derived in Refs. $[19,20]$. This criterion states that spiral waves destabilize when the wave trains emitted by the spiral waves become absolutely unstable which, in turn, occurs when a branch point $\lambda_{\mathrm{bp}}$ of their linear dispersion relation, given by $\lambda_{\mathrm{bp}}=\lambda_{*}(\nu)$ with $\mathrm{d} \lambda_{*} / \mathrm{d} \nu(\nu)=0$, crosses the imaginary axis. Our results show that point eigenvalues caused by curvature effects may destabilize spirals prior to branch points crossing and that the occurrence of these eigenvalues can be predicted from the asymptotic profile of the spiral.

\section{APPLICATIONS}

First, we compare the prediction in Eq. (18) with the spectral computations by Barkley and Wheeler [13], reproduced in Fig. 3, for the modified FitzHugh-Nagumo equation

$$
\begin{aligned}
u_{t} & =\Delta u-\frac{1}{\epsilon} u(u-1)\left(u-\frac{b+v}{a}\right), \\
v_{t} & =f(u)-v
\end{aligned}
$$

with $f(u)$ as in Ref. [6] and parameters as in Ref. [14, (14)-(15)]. Note that our results are applicable to Eq. (20) even though one of its diffusion coefficients vanishes. Indeed, the $v$-component of the eigenvalue problem associated with a spiral wave $\left(u_{*}, v_{*}\right)$ of Eq. (20) is given 


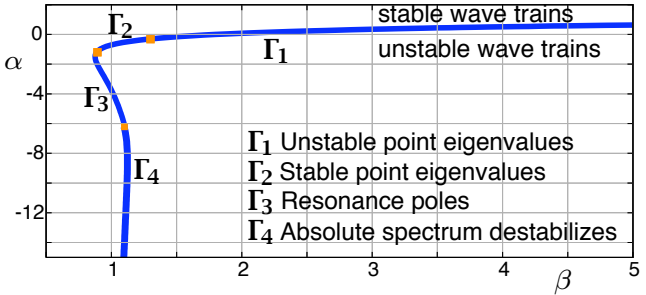

FIG. 5: The solid curve, shown in the $(\alpha, \beta)$-plane and divided into curve segments $\Gamma_{j}$, is the absolute instability limit of the wave trains selected by spiral waves of the complex GinzburgLandau equation: The wave trains are absolutely unstable below the solid curve and convectively unstable above it. See text for further details.

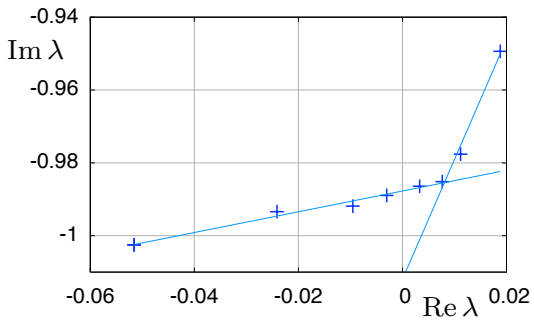

FIG. 6: The critical part of the spectrum of the spiral of Eq. (21), computed on a disk of radius $R=140$, is shown for $(\alpha, \beta)=(0.54,4.2) \in \Gamma_{1}$. The branch point of the dispersion relation is given by $\lambda_{\mathrm{bp}}=(0,-0.99)$.

in the corotating frame by

$$
\left(\lambda+1-\omega \partial_{\varphi}\right) v=f_{u}\left(u_{*}\right) u
$$

The operator on the left-hand side can be inverted for each $\lambda$ with $\operatorname{Re} \lambda>-1$ and substituted into the equation for $u$, resulting in a nonlocal eigenvalue problem for $u$ with a non-zero diffusion coefficient. The remaining analysis proceeds then exactly as before.

The absolute spectra of the spiral wave of Eq. (20) have been previously computed in Ref. [14] which allows us to compute $d$, while the coefficient $a$ can be calculated as outlined at the end of Section III using the boundary-value problem solver AUTO [21]. Substitution into Eq. (18) gives a prediction for the asymptotic angle of the ray, emanating from the branch point, along which the eigenvalues in Fig. 3 to the right of the absolute spectrum should align themselves. We also estimated this angle from Fig. 3 using the branch point at the edge of the absolute spectrum as origin. The resulting values are

\begin{tabular}{|l||c||c|}
\hline Breakup at: & core & far field \\
\hline $\arg (a / d)$ computed with AUTO & $40^{\circ}$ & $44^{\circ}$ \\
\hline $\arg \Lambda_{n}$ extracted from Ref. [13] & $48^{\circ}$ & $45^{\circ}$ \\
\hline
\end{tabular}

which agree quite reasonably given that there are only one or two point eigenvalues visible in Fig. 3.

Next, we consider spiral waves of the planar complex Ginzburg-Landau equation

$$
A_{t}=(1+\mathrm{i} \alpha) \Delta A+A-(1+\mathrm{i} \beta)|A|^{2} A
$$

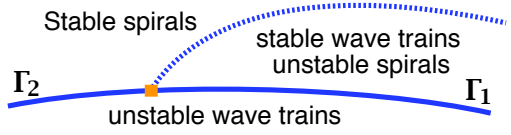

FIG. 7: A schematic illustration of the instability region of spiral waves in $(\alpha, \beta)$-parameter space. The dotted curve corresponds to those parameter values for which the first point eigenvalue of the spiral wave crosses the imaginary axis.

on $B_{R}(0)$ with Neumann boundary conditions. Spiral waves of Eq. (21) satisfy an ODE and can therefore be computed using AUTO.

We have calculated the curve in $(\alpha, \beta)$-space along which the absolute spectrum of the asymptotic wave trains crosses the imaginary axis, leading to an absolute instability: This curve, further divided into the four curve segments $\Gamma_{1}, \ldots, \Gamma_{4}$ which will be explained shortly, is shown in Fig. 5. Our computations show that the absolute spectrum crosses at a branch point for each $(\alpha, \beta)$ on one of the curve segments $\Gamma_{1}, \Gamma_{2}$ and $\Gamma_{3}$, thus confirming the results in Ref. [20]. However, for $(\alpha, \beta)$ on the curve segment $\Gamma_{1}$, the spiral wave is already unstable due to point eigenvalues which have crossed the imaginary axis prior to the branch point: our numerical computations show that both $\Phi$ from Eq. (16) and $\Psi$ from Eq. (18) are smaller than $\pi / 2$ for parameters on $\Gamma_{1}$. We computed the point eigenvalues that arise due to our results in $\S \mathrm{III}$ and $\S \mathrm{IV}$ for $(\alpha, \beta)=(0.54,4.2) \in \Gamma_{1}$ and plotted them in Fig. 6 . Along the curve $\Gamma_{2}$, the point eigenvalues are also present but lie to the left of the imaginary axis since the angle $\Psi$ from Eq. (18) is larger than $\pi / 2$. Lastly, along the curve segment $\Gamma_{3}$, the angle $\Phi$ is larger than $\pi / 2$ so that there are no point eigenvalues emerging from the branch point of the linear dispersion relation. Thus, in summary, our results show that the stability region for spiral waves in the complex GinzburgLandau equation is smaller than that predicted by the absolute spectrum (see Fig. 7 for a schematic illustration).

For completeness, we point out that along the curve segment $\Gamma_{4}$ the absolute spectrum crosses the imaginary axis away from branch points. In fact, the linear dispersion relation of the spiral does not have any branch points with the correct Morse index, and the criterion given in Ref. [20] therefore fails to predict the correct onset of instability.

\section{CONCLUSIONS}

We showed that the linearization about spiral waves near branch points can be reduced to a non-selfadjoint Schrödinger operator with a complex long-range potential which accounts for curvature effects and decays algebraically like $1 / r$ in the radius $r$. The spectral properties of the Schrödinger operator are characterized by two complex coefficients $a$ and $d$. Based on this reduc- 
tion, we have provided theoretical evidence that spiral spectra contain isolated point eigenvalues near branch points whenever the angle $\Phi=\arg (a / d)$ has modulus less than $\pi / 2$. The number of these eigenvalues increases as the domain size increases. Furthermore, if the angle $\Psi=\arg \left(a^{2} / d\right)$ has modulus less than $\pi / 2$, then these point eigenvalues destabilize the spiral prior to an absolute instability. It is worthwhile to mention that branch points of spiral waves are periodic in the imaginary direction with period given by the angular velocity $\omega$ : eigenvalues will emerge simultaneously from all these periodically spaced branch points.

Our results have the following consequence. A standard test for absolute instabilities of spirals is the branch point or saddle-point criterion due to Refs. [19, 20] which states that absolute instabilities of spirals occur whenever certain branch points of their linear dispersion relation cross the imaginary axis. As shown in this paper, point eigenvalues emerging from branch points may destabilize spirals prior to branch points crossing. The location of these eigenvalues can be predicted from the angles $\Phi$ and $\Psi$ through the coefficients $a$ and $d$ using only the asymptotic 1D profile of the spiral. We showed that this instability scenario occurs for a modified FitzHugh-Nagumo equation and the complex Ginzburg-Landau equation.

We thank D. Barkley and P. Wheeler for interesting discussions, sharing an early version of Ref. [13], and granting permission to use Fig. 3. We gratefully acknowledge support from the NSF and the Royal Society.
[1] A. T. Winfree, When Time Breaks Down, (Princeton University Press, Princeton, 1987); R. Kapral and K. Showalter (Eds.), Chemical Waves and Patterns, (Kluwer, Doordrecht, 1995); J. Keener and J. Sneyd, Mathematical Physiology, (Springer, Berlin, 1998).

[2] W. Jahnke, W. E. Skaggs, and A. T. Winfree, J. Chem. Phys. 93, 740 (1989); T. Plesser, S. C. Müller, and B. Hess, J. Phys. Chem. 94, 7501 (1990); G. S. Skinner and H. L. Swinney, Physica D 48, 1 (1991); V. PerezMunuzuri, R. Aliev, B. Vasiev, V. Perez-Villar, and V. I. Krinsky, Nature 353, 740 (1991); G. Li, Q. Ouyang, V. Petrov, and H. L. Swinney, Phys. Rev. Lett. 77, 2105 (1996); L. Q. Zhou and Q. Ouyang, Phys. Rev. Lett. 85, 1650 (2000).

[3] Focus Issue: Fibrillations in Normal Ventricular Myocardium, Chaos 8(1) (1998).

[4] S. Nettesheim, A. von Oertzen, H. H. Rotermund, and G. Ertl, J. Chem. Phys. 98, 9977 (1993).

[5] D. Barkley, Phys. Rev. Lett. 68, 2090 (1992); Phys. Rev. Lett. 72, 164 (1994).

[6] M. Bär and M. Eiswirth, Phys. Rev. E 48, 1635 (1993).

[7] Q. Ouyang and J. M. Flesselles, Nature 379, 143 (1996).

[8] M. Bär and M. Or-Guil, Phys. Rev. Lett. 82, 1160 (1999).

[9] M. Yoneyama, A. Fujii and S. Maeda, J. Am. Chem. Soc. 117, 8188 (1995).

[10] A. Goryachev, H. Chaté and R. Kapral, Phys. Rev. Lett.
80, 873 (1998).

[11] A. Hagberg and E. Meron, Chaos 4, 477 (1994).

[12] J.-M. Chomaz and A. Couairon, Phys. Fluids 11, 2977 (1999); Physica D 108, 236 (1997); S. M. Tobias, M. R. E. Proctor and E. Knobloch, Physica D 113, 43 (1998); B. Sandstede and A. Scheel, J. Diff. Eqns. 208, $176(2005)$.

[13] D. Barkley and P. Wheeler, preprint, (2005).

[14] B. Sandstede and A. Scheel, Phys. Rev. E 62, 7708 (2000).

[15] B. Sandstede and A. Scheel, Physica D 145, 233 (2000).

[16] M. Reed and B. Simon, Methods of Modern Mathematical Physics IV,(Academic Press, San Diego, 1978).

[17] L. J. Slater, Confluent Hypergeometric Functions, (Cambridge University Press, Cambridge, 1960).

[18] F. W. J. Olver, Asymptotics and Special Functions, (Academic Press, New York, 1974).

[19] R. J. Briggs, Electron-Steam Interaction With Plasmas, (MIT Press, Cambridge, 1964).

[20] I. S. Aranson, L. Aranson, L. Kramer, and A. Weber, Phys. Rev. A 46, 2992 (1992).

[21] E. J. Doedel, A. R. Champneys, T. F. Fairgrieve, Yu. A. Kuznetsov, B. Sandstede, and X. Wang, Technical Report, Concordia University, 1997. 